\title{
Risk factors predicting development of complications in 72 dogs with esophageal foreign bodies
}

\author{
Bohye Shin, Hakhyun Kim, Dongwoo Chang, Ji-Houn Kang*, Byeong-Teck Kang, Mhan-pyo Yang \\ Veterinary Medical Center, College of Veterinary Medicine, Chungbuk National University, Cheongju 28644, Korea
}

\begin{abstract}
The aim of this study was to determine predictive risk factors implicated in complications in dogs with esophageal foreign bodies. Medical records of 72 dogs diagnosed with esophageal foreign bodies by endoscopy were reviewed retrospectively. Factors analyzed included age; breed; gender; body weight, location, dimension, and type of foreign body; and duration of impaction. To identify risk factors associated with complications after foreign body ingestion, categorical variables were analyzed using the chisquare or Fisher's exact tests and multivariate analysis, as appropriate. Complications secondary to esophageal foreign body ingestion included megaesophagus, esophagitis, perforation, laceration, diverticulum, and pleuritis. Univariate analysis revealed that the location and duration of impaction after foreign body ingestion were associated with an increased risk of esophageal laceration and perforation. Multivariate analysis showed that age, duration of impaction, and foreign body dimension were significant independent risk factors associated with the development of complications in dogs with esophageal foreign bodies. In conclusion, these results showed that longer duration of impaction and larger foreign body dimensions may increase the risks of esophageal laceration, perforation, and plueritis in dogs.
\end{abstract}

Keywords: endoscopy, esophagitis, esophageal diverticulum, esophageal perforation, pleuritis

\section{*Corresponding author}

\section{Ji-Houn Kang}

Veterinary Medical Center, College of Veterinary Medicine, Chungbuk National University, 1 Chungdae-ro, Seowon-gu, Cheongju 28644, Korea

Tel: $+82-43-261-3691$

Fax: +82-43-269-2595

E-mail: jhkang@chungbuk.ac.kr

ORCID:

Ji-Houn Kang,

https://orcid.org/0000-0001-8536-3027

Conflict of Interest

The authors declare no conflicts of interest.

Received: November 30, 2018

Revised: January 23, 2019

Accepted: February 25, 2019

\section{Introduction}

Small animlas that ingest esophageal foreign bodies (EFBs) frequently require emergency treatments in small animal practice [1-6]. The esophageal walls are composed of the mucosa, submucosa, muscularis, and adventitia [7]. Some materials too large to pass through the esophagus may become embeded and abrade the esophageal walls. Consequently, EFBs can cause severe complications such as perforation and laceration of the esophageal walls [810]. These complications can lead to a life-threatening conditions including pleuritis. Additionally, because the esophagus lacks a serosal layer, unlike other gastrointestinal organs in dogs, early fibrin sealing of esophagostomy regions may occur more slowly [7]. Therefore, EFBs should be considered an emergency condition in dogs, requiring prompt removal.

Several studies addressed EFBs in dogs [11-13], and all emphasized that EFBs are likely to cause critical complications. However, there is still a lack of information available on factors associated with the development of complications, and few of these studies focused on endoscopic procedures in dogs with EFBs. Therefore, the objective of the present study was to determine risk factors predictive of complications in dogs with EFBs diagnosed after endoscopic retrieval.

\section{Materials and Methods}

The medical records of our institution were retrospectively reviewed to identify dogs treated for EFBs between October 2010 and August 2016. Animals were included only if foreign bodies became wedged into the esophagus. Dogs were excluded if foreign bodies were impacted both the stomach and esophagus; if foreign bodies were present in other gastrointestinal organs, the nasal cavity, or the ear canal; or if EFBs were pushed into the stomach or retrieved surgically. Animals were also excluded if EFBs were resolved with- 
out intervention, if owners declined treatment, or if dogs had concurrent diseases or a possibility of other diseases, or if medical and follow-up records were incomplete.

Clinical signs were described by the owner or recorded by the clinician at physical examination. The clinical decision to perform endoscopy was based on history, clinical signs, and interpretation of radiographs. All radiographs were reviewed by a professional radiologist. Dogs were finally diagnosed to EFB if endoscopy detected a foreign body in the esophagus. EFB were subsequently removed using a flexible gastroscope (Ricoh imaging company LTD., Japan), together with grasping forceps, retrieval baskets, and/or snares. Grasping forceps were widely used and were the first choice for retrieval of all types of EFBs. Retrieval baskets and snares were also considered if necessary. To confirm impairment of esophagus, the lumen of the esophagus was endoscopically re-evaluated after retrieval of the EFB. Two veterinarians independently described the type and dimensions of each foreign body, its location in the esophagus, and any resulting complications.

Pre-treatment evaluation included medical history, the results of physical examinations, indicators of EFB impaction, and reactions to anesthesia. Clinicopathologic tests to determine the possibility of anesthesia included complete blood counts and serum biochemical profile. Airway management was considered critical during endoscopic procedure. All dogs were subcutaneously injected with $1 \mathrm{mg} / \mathrm{kg}$ maropitant citrate (Zoetis Inc., USA) followed by intramuscular injection of $0.04 \mathrm{mg} / \mathrm{kg}$ atropine sulfate (Jeil Pharmaceutical Co. Ltd., Korea) 15-20 minutes prior to the induction of anesthesia. Anesthesia was induced with propofol (Myungmoon Pharm. Co., Ltd., Korea), consisting initially of intravenous injections of boluses of $1 / 4$ to $1 / 3$ of the original propofol dose (4 to $6 \mathrm{mg} / \mathrm{kg}$ ) followed by slow injection of the remaining propofol if dogs did not reach suitable anesthetic levels with the bolus doses. Anesthesia during endoscopic procedures was maintained with isoflurane (Piramal Critical Care Inc., USA). Duration of anesthesia was defined as the time from the induction of anesthesia to the end of inhalation.

Dogs were positioned in left lateral recumbent position during endoscopy. After endoscopic retrieval of the EFBs, the presence or absence of complications and types of complications, including the development of esophageal strictures, were evaluated endoscopically.

Records of all dogs at the time of EFB diagnosis were reviewed. Factors analyzed included gender, body weight, age, breed, and clinical signs. Duration of impaction was defined as the time from symptom onset to the end of the endoscopic procedure. The location of EFBs was determined by radiography and endoscopy and was classified as being in the cervical esophagus, the cranial esophagus at the base of the heart, and the caudal esophagus between the base of the heart and the diaphragmatic hiatus. The size of removed foreign bodies were evaluated by measuring the longest trans- verse and longitudinal dimensions. If EFBs had been removed piecemeal, their dimensions were evaluated radiographically during endoscopy.

The presence of complicated lesions in the esophageal lumen was confirmed as described [4]. Briefly, diffuse esophageal dilation and aperistalsis were confirmed as being megaesophagus, as determined by thoracic radiography. Esophagitis was defined as circular and confluent erosion. Laceration was defined as the progression of esophagitis-associated bleeding from tears in the mucosa. Esophageal diverticulum was defined as a saclike outpouching of the esophageal lumen containing impacted ingesta, together with localized esophagitis. Esophageal perforation was identified radiographically with contrast media or by computed tomography. Pleural effusion was initially diagnosed by thoracic radiography, with positive bacterial culture of the pleural effusion confirming pleuritis. Esophageal stricture in dogs with impeded food passage down the esophageal lumen was confirmed by follow-up endoscopy.

\section{Statistical analyses}

Risk factors including age ( $\leq 1$ vs. $2-6$ vs. $\geq 7$ years), body weight ( $\leq 3.03$ vs. $>3.03 \mathrm{~kg}$ ), breed (Pekingese, Shih Tzu, Pomeranian, Yorkshire Terrier, Maltese, Miniature Poodle, Chihuahua, and eight other breeds), sex (intact male vs. neutered male vs. intact female vs. spayed female), EFB location (cervical vs. cranial vs. caudal esophagus), type of foreign bodies (fruit vs. rawhide vs. bone vs. linear type), impaction time ( $\leq 24$ vs. $>24 \mathrm{~h}$ ), foreign body dimension $(\leq 8.06$ vs. $>8.06 \mathrm{~cm}^{2}$ ), and complications (megaesophagus vs. esophagitis vs. laceration vs. diverticulum) were examined. All percentages were rounded to one decimal place except for incidence of EFBs.

To identify the risk factors associated with the development of complications after foreign body ingestion, dogs were divided into those with and without complications were evaluated using Pearson's $\chi^{2}$ test or Fisher's exact test, as appropriate. Factors with a value of $p<0.2$ in univariate analyses were selected for the initial multivariable model. Significant variables identified by univariate analyses were further analyzed by multivariate logistic regression analysis (enter method) with the level of significance defined as $p<0.05$. In logistic regression, five dogs with linear types of foreign bodies were excluded from all categories. A commercially available statistical software program (IBM Co., USA) was used for data analysis.

\section{Results}

A review of the medical records of our institution identified 172 dogs diagnosed with EFBs between October 2010 and August 2016. Of these, 65 dogs were excluded, including 49 treated with surgical retrieval, 12 that showed spontaneous resolution, and four whose owner declined treatment. Of the 105 remaining dogs that underwent endoscopic removal 
Table 1. Characteristics of 72 dogs with esophageal foreign bodies

\begin{tabular}{lc}
\hline \multicolumn{1}{c}{ Variables } & $\begin{array}{c}\text { Number of dogs with } \\
\text { EFBs }(\mathrm{n}=72)\end{array}$ \\
\hline Gender & 9 \\
Males & 26 \\
Neutered males & 8 \\
Females & 29 \\
Spayed females & $3.03(1.09 .5)$ \\
Body weight (kg), median (range) & 36 \\
$\leq 3.03$ & 36 \\
$>3.03$ & $5.9(19)$ \\
Age (yr), median (range) & 9 \\
$\leq 1$ & 38 \\
$2-7$ & 25 \\
$\geq 7$ & \\
Breed & 23 \\
Maltese & 15 \\
Yorkshire terrier & 9 \\
Shih Tzu & 8 \\
Pomeranian & 4 \\
Miniature Poodle & 3 \\
Pekingese & 2 \\
Chihuahua & 8 \\
Other breeds & \\
\hline
\end{tabular}

EFB, esophageal foreign body.

of foreign bodies, 19 had incomplete medical and follow-up records and 16 had concurrent disorders, such as chronic kidney disease, myxomatous mitral valve disease, diabetes mellitus, meningioma, intervertebral disc disease, hyperadrenocorticism, and hypoadrenocorticism. Finally, 72 dogs met the criteria for this study.

These 72 dogs included 35 males (26 neutered, 9 intact) and 37 females (29 spayed, 8 intact) (Table 1). The median age of these dogs was 5.90 years, with a range of 2 months to 15 years. Nine dogs $(12.5 \%)$ were less than 1-year-old at presentation, $38(52.8 \%)$ were aged between 1 and 7 years, and $25(34.7 \%)$ were aged more than 7 years. Their median bodyweight was $3.03 \mathrm{~kg}$ (range, 1.02-9.50 kg), with 36 each weighing $\leq 3.03 \mathrm{~kg}$ and $>3.03 \mathrm{~kg}$. These 72 dogs belonged to 15 breeds, including. 23 Maltese, 15 Yorkshire Terrier, nine Shih Tzu, eight Pomeranian, four Miniature Poodle, three Pekingese, two Chihuahua, and one each of following eight breeds: Miniature Schnauzer, West Highland White Terrier, Cocker Spaniel, Japanese Spitz, Boston Terrier, Pug, Chinchilla, and Mongrel.

Clinical signs at presentation were included vomiting $(n=42)$, retching $(n=23)$, lethargy $(n=13)$, anorexia $(n=10)$, gagging $(n=9)$, dullness $(n=4)$, and drooling $(n=2)$. All 72 dogs had more than one clinical sign, and 37 had more than two. EFBs were suspected on radiographs in 67 of the 72 dogs, whereas five dogs underwent direct endoscopic evalua-
Table 2. Complications observed in 38 dogs with esophageal foreign bodies

\begin{tabular}{lc}
\hline \hline \multicolumn{1}{c}{ Complications $(\mathrm{n}=38)$} & $\begin{array}{c}\text { Number of dogs } \\
\text { with complications }(\%)\end{array}$ \\
\hline Esophagitis & $21(55.3)$ \\
Megaesophagus & $9(23.7)$ \\
Esophageal diverticulum & $6(15.8)$ \\
Esophageal laceration & $5(13.2)$ \\
Esophageal perforation & $3(7.9)$ \\
Pleuritis & $3(7.9)$ \\
\hline
\end{tabular}

Table 3. Univariate analysis of risk factors for complications in the 72 dogs with esophageal foreign bodies

\begin{tabular}{|c|c|c|c|}
\hline Variables & $\begin{array}{c}\text { Dogs without } \\
\text { complications } \\
(\mathrm{n}=34)\end{array}$ & $\begin{array}{l}\text { Dogs with } \\
\text { complications } \\
(\mathrm{n}=38)\end{array}$ & $p$ value \\
\hline Gender & & & 0.803 \\
\hline Males & 16 & 19 & \\
\hline Females & 18 & 19 & \\
\hline Body weight $(\mathrm{kg})$ & & & 0.863 \\
\hline$\leq 3.03$ & 15 & 21 & \\
\hline$>3.03$ & 19 & 17 & \\
\hline Age & & & $<0.001$ \\
\hline$\leq 1$ & 8 & 1 & \\
\hline $2-7$ & 21 & 17 & \\
\hline$\geq 7$ & 5 & 20 & \\
\hline Location & & & 0.173 \\
\hline Cervical esophagus & 2 & 0 & \\
\hline Cranial esophagus & 14 & 8 & \\
\hline Caudal esophagus & 18 & 30 & \\
\hline Duration of impaction (h) & & & $<0.001$ \\
\hline$\leq 24$ & 24 & 12 & \\
\hline$>24$ & 10 & 26 & \\
\hline Type of foreign body & & & 0.357 \\
\hline Fruit & 4 & 5 & \\
\hline Rawhide & 19 & 15 & \\
\hline Bone & 8 & 16 & \\
\hline Linear type & 3 & 2 & \\
\hline $\begin{array}{l}\text { Dimension of foreign } \\
\text { body }\left(\mathrm{cm}^{2}\right)\end{array}$ & & & $<0.001$ \\
\hline$\leq 8.06$ & 22 & 11 & \\
\hline$>8.06$ & 9 & 25 & \\
\hline Linear type & 3 & 2 & \\
\hline
\end{tabular}

tion without radiographic suspicion of the presence of EFBs.

Complications were observed in 38 of the 72 dogs (Table 2). Esophagitis was the most frequent complications, being observed in 30 of 38 dogs with complications (78.9\%). Other EFB-associated complications included megaesophagus $(\mathrm{n}=9$, $23.7 \%)$, esophageal diverticulum $(n=6,15.8 \%)$, and laceration $(\mathrm{n}=5,13.2 \%)$ and esophageal perforation and pleuritic $(\mathrm{n}=3,7.9 \%)$. 
Bohye Shin, Hakhyun Kim, Dongwoo Chang, Ji-Houn Kang, Byeong-Teck Kang, Mhan-pyo Yang

Table 4. Multivariate analysis of risk factors for complications in $72 \mathrm{dogs}$ with esophageal foreign bodies

\begin{tabular}{lccc}
\hline \multicolumn{1}{c}{ Variables } & OR & $95 \%$ CI & $p$ value \\
\hline Age $\geq 7$ yr (vs. $<7$ yr) & 17.946 & $1.246-258.095$ & 0.034 \\
Location of caudal esophagus (vs. no caudal esophagus) & 1.475 & $0.070-30.959$ & 0.802 \\
Duration of impaction $>24 \mathrm{~h}$ (vs. $\leq 24 \mathrm{~h}$ ) & 4.716 & $1.295-17.183$ & 0.019 \\
Dimension of EFB $>8.06 \mathrm{~cm}^{2}$ (vs. $\leq 8.06 \mathrm{~cm}^{2}$ ) & 4.667 & $1.284-16.966$ & 0.019 \\
\hline
\end{tabular}

CI, confidence interval; EFB, esophageal foreign body; OR, odds ratio.

Table 5. Clinical characteristics of seven dogs with esophageal laceration, perforation, and/or pleuritis caused by esophageal foreign bodies

\begin{tabular}{|c|c|c|c|c|c|c|c|}
\hline Complications & Breed & Gender & Age (yr) & Location & $\begin{array}{l}\text { Duration of } \\
\text { impaction }(\mathrm{h})\end{array}$ & $\begin{array}{l}\text { Dimension of } \\
\text { EFBs }\left(\mathrm{cm}^{2}\right)\end{array}$ & $\begin{array}{l}\text { Type of } \\
\text { EFBs }\end{array}$ \\
\hline Laceration & Mongrel & Spayed female & 6 & $\begin{array}{l}\text { Caudal } \\
\text { esophagus }\end{array}$ & 6 & 12.8 & Bone \\
\hline Laceration & Maltese & Spayed female & 5 & $\begin{array}{l}\text { Caudal } \\
\text { esophagus }\end{array}$ & 120 & 6.12 & $\begin{array}{l}\text { Rawhide } \\
\text { (Sausage) }\end{array}$ \\
\hline Laceration & Yorkshire Terrier & Neutered male & 9 & $\begin{array}{l}\text { Caudal } \\
\text { esophagus }\end{array}$ & 240 & 10.5 & Bone \\
\hline Laceration & Japanese Spitz & Female & 2 & $\begin{array}{l}\text { Caudal } \\
\text { esophagus }\end{array}$ & 96 & 10.5 & Bone \\
\hline Perforation and pleuritis & Maltese & Spayed female & 11 & $\begin{array}{l}\text { Caudal } \\
\text { esophagus }\end{array}$ & 72 & ND & Linear type \\
\hline Perforation and pleuritic & Pomeranian & Neutered male & 4 & $\begin{array}{l}\text { Caudal } \\
\text { esophagus }\end{array}$ & 192 & 8 & Bone \\
\hline $\begin{array}{l}\text { Laceration, perforation, } \\
\text { and pleuritis }\end{array}$ & White Terrier & Female & 3 & $\begin{array}{l}\text { Caudal } \\
\text { esophagus }\end{array}$ & 120 & 11.2 & $\begin{array}{l}\text { Rawhide } \\
\text { (Sausage) }\end{array}$ \\
\hline
\end{tabular}

EFB, esophageal foreign body; ND, not determined.

Univariate analysis showed that several factors differing significantly in dogs with and without complications, including age $(p<0.001)$, EFB location $(p=0.173)$, duration of impaction $(p<0.001)$, and EFB dimensions $(p<0.001)$ (Table $3)$. However, there were no significant differences in the gen$\operatorname{der}(p=0.803)$, body weight $(p=0.863)$, and EFB types ( $p=$ $0.357)$.

Of the 72 dogs, 48 (66.7\%) had EFBs in the caudal portion of the esophagus, with $30(62.5 \%)$ of these $48 \mathrm{dogs}$ developing complications (Table 3$)$. Only two dogs $(2.8 \%)$ had EFBs in the cervical portion of the esophagus, with neither having complications. The remaining 22 dogs (30.6\%) had EFBs in the cranial part of the esophagus, with 14 (63.6\%) of these 22 dogs having complications.

Of the 72 dogs, 36 were evaluated within the first $24 \mathrm{~h}$ after symptom onset, whereas the other 36 had clinical signs for more than $24 \mathrm{~h}$ prior to evaluation (Table 3 ). The mean duration of impaction was about 19 hours. Commonly ingested objects were pieces of rawhide $(n=34,47.2 \%)$, including sausages, dental chews, snacks for dogs, sweet potatoes, and potatoes; bones $(n=24,33.3 \%)$ from chicken, pork, and beef; fruit $(n=9,12.5 \%)$, such as apples, pineapples, and pears; or linear objects $(n=5,6.9 \%)$, such as thread, needles, and plant roots.

Of the 72 dogs, $33(45.8 \%)$ ingested EFBs $\leq 8.06 \mathrm{~cm}^{2}$ in size, with $11(33.3 \%)$ of these 33 dogs developing complications. Thirty-four dogs $(47.2 \%)$ had EFBs $>8.06 \mathrm{~cm}^{2}$, with $25(73.5 \%)$ of these 34 dogs developing complications. Linear foreign bodies were lodged into the esophagus of five dogs $(6.9 \%)$ and were not evaluated.

Multivariate logistic analysis showed that older age (odds ratio $[\mathrm{OR}], 17.946 ; 95 \%$ confidence interval [CI], 1.24$258.09 ; p=0.034)$, longer duration of impaction (OR, 4.716; 95\% CI 1.29-17.18; $p=0.019)$ and increased dimension of EFBs (OR, 4.667; 95\% CI 1.28-16.96; $p=0.019$ ) were significant independent risk factors associated with the development of complications in dogs with EFBs (Table 4).

Seven (19.4\%) of 36 dogs had esophageal laceration, perforation, and/or pleuritic (Table 5), with EFBs in all seven of these dogs being located in the caudal esophagus. The mean size of EFBs in these seven dogs was $9.9 \mathrm{~cm}^{2}$, and their mean duration of impaction was 120.9 hours. Three (43\%) of these seven dogs showed pleuritic; all were hospitalized in intensive care and discharged within 10 days. Two representative cases were presented (Figs. 1 and 2); bony materials in one induced localized perforation (representative case 1), whereas obstructions by sausages containing water-insoluble materials triggered severe hemorrhagic lesion throughout the entire esophagus (representative case 2). All of these lesions progressed to esophageal stricture. 

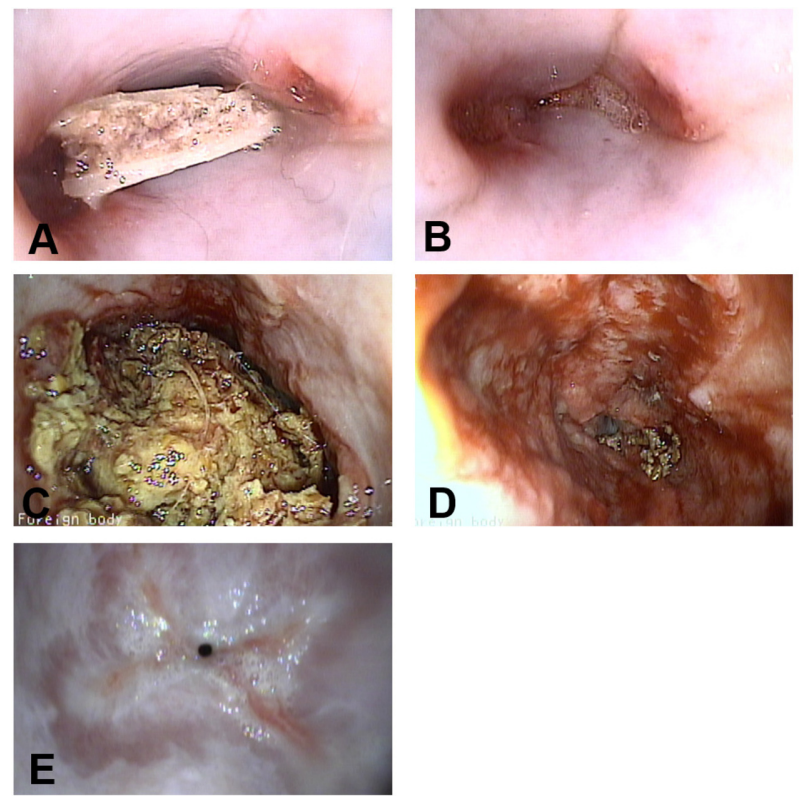

Fig. 1. Representative endoscopic images of the esophagus before (A and $\mathrm{C})$ and after (B, D, and $\mathrm{E}$ ) endoscopic retrieval of foreign bodies in two representative cases. In case 1, (A) Bone obstruction visualized by endoscopy. (B) After endoscopic retrieval, perforation and laceration were present at the site the foreign body was lodged. In case 2, (C) Water-insoluble sausage visualized by endoscopy. (D) After endoscopic retrieval, severe laceration and perforation were shown throughout the entire esophageal wall, (E) with follow-up endoscopy showing an esophageal stricture.
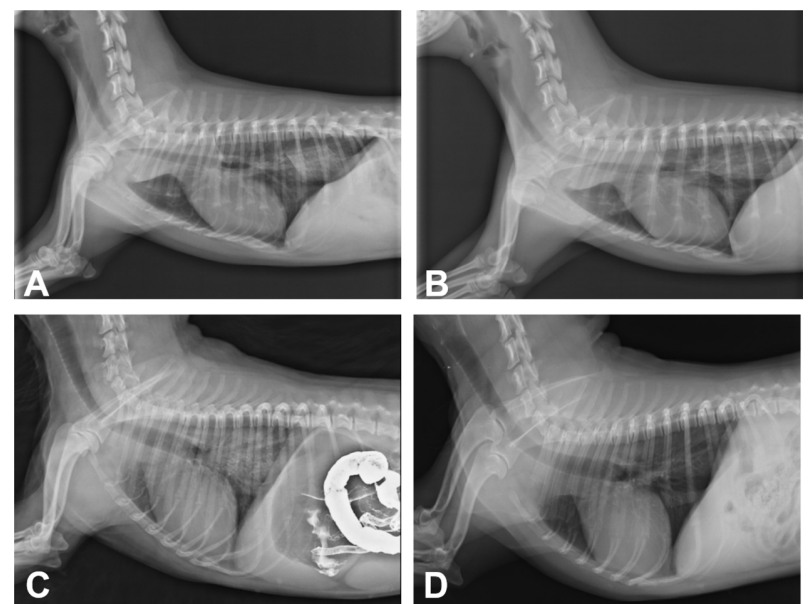

Fig. 2. Thoracic radiographs of the esophagus before and after endoscopic retrieval of foreign bodies in representative case 1 (A and $\mathrm{B}$ ) and 2 (C and $\mathrm{D})$.

\section{Discussion}

Our results showed that, in dogs with EFBs, the development of complications was significantly associated with older age ( $\geq 7$ years), longer duration of impaction (above $24 \mathrm{~h}$ ), and larger EFB dimensions $\left(>8.06 \mathrm{~cm}^{2}\right)$. By contrast, gen- der, body weight, type of EFBs, and EFB location were not associated with complications. We also found that EFBs were frequently in dogs with bony type foreign bodies. Although type of EFBs was not risk factor, sausage which was soft and fragile material could induce severe complication such as esophageal perforation and pleuritis. Previous studies also showed that bone obstruction is associated with a relatively high complication rate in dogs $[1,6,11-15]$. The sharp edges of bony type of foreign bodies can easily damage the esophageal muscular layer, whereas foreign bodies with smooth edges, such as sausages, cannot. Nevertheless, despite the absence of sharp edges, sausages of relatively large dimension lodged in the esophagus for over $24 \mathrm{~h}$ can induce the development of severe complications such as esophageal laceration. A longer duration of EFBs impaction is associated with a poorer outcome, which might be explained by the presumption that prolonged EFB impaction would be more likely to cause esophageal ulceration or perforation [16]. In addition, blunt objects impacted in the esophagus that are causing esophageal perforation may be the result ongoing ischemic pressure injury [1]. In the present study, esophageal strictures developed in two dogs presenting with severe lacerations caused by a prolonged sausages entrapment (duration of impaction $=120 \mathrm{~h}$ ). Our results indicate the duration of impaction are important in predicting the development of complications whether sharp edges of EFBs or not. The duration of clinical signs before presentation was reported to be significantly shorter in dogs with mild than with moderateto-severe esophagitis [12], and the duration of hospitalization of dogs with EFBs may be dependent on the duration of clinical signs prior to presentation [13]. In humans, the duration of impaction differed significantly between patients with and without complications [17]. Taken together, these findings emphasize the importance of prompt removal of EFBs [18].

We also found that age was a risk factor for the development of complications. By contrast, a previous study in dogs with EFBs showed that age did not differ significantly between dogs with the mild and moderate-to-severe esophagitis [12], although there was no statistical analysis on the development of complications. In human medicine, age has been associated with a deterioration of esophageal function, including increased stiffness and reduced primary and secondary peristalsis [19]. EFBs are ingested by swallowing and lodged in the esophagus, a muscular tube leading from the mouth to the stomach [7]. First peristalsis is stimulated by the oropharyngeal phase, whereas food within the esophagus stimulates secondary peristalsis [20]. If EFBs are lodged in the esophagus, peristalsis will be reduced or eliminated, especially in older dogs, resulting in a refractory period [20]. Injuries may extend beyond the mucosal layer. Although reported to date only in human esophagus, peristalsis may be enervated in older dogs, resulting in increased esophageal stiffness. Therefore, enervated muscles may easily damage the mucosa, resulting in severe complications. Although this study included only dogs without other diseases, concealed 
concurrent disorders may increase with age. Moreover, age was not a risk factor for comlications in humans with EFBs [17]. To our knowledge, no study to date has evaluated the correlation between age and esophageal muscle weakness in dogs. Further studies are therefore warranted.

We also observed a significant difference in the dimensions of EFBs between dogs with and without complications. Although a previous study reported no significant association between length of hospitalization and EFBs [13], that study evaluated EFBs dimensions. Moreover, EFBs dimensions differed significantly between human patients with and without complications [17].

The most commonly affected region was the caudal esophagus, between the base of the heart and the diaphragmatic hiatus. However, we found no correlation between EFB location and the development of complications. Some studies in dogs showed that most EFBs were located in the caudal esophagus [11, 13, 14, 21-23]. One study reported that the location of EFBs and gastric foreign bodies in dogs was not a risk factor for complications or death/euthanasia [21], whereas another reported that EFB location in the caudal esophagus was associated with a longer hospital stay [13]. A study showing that almost all EFBs were located in the caudal esophagus found no significant difference in EFB location between subjects that underwent successful and unsuccessful endoscopy [13]. EFB location was not a risk factor for the development of complications in humans [17]. Physical narrowing of the esophagus is likely to impact foreign bodies. Despite our finding that location was not a risk factor for complications, we observed that all dogs with laceration, perforation and/or pleuritis had EFBs in the caudal esophagus. The lack of association between EFB location and complications may have been due to small sample size, suggesting the need for further investigations.

Clinical signs of EFB impaction in the present study included vomiting, retching, lethargy, anorexia, gagging, and hypersalivation and were similar to those observed in previous reports [13, 21, 24]. None of these clinical signs differed significantly in dogs that did and did not develop complications. This result, however, was somewhat reflected in the clinicians' diagnostic approaches. Studies reported that severity of esophageal damage was depended on the severity of clinical signs $[12,25]$. Many of the dogs we evaluated were toy breeds, which reflects the distinct characteristics of the area in which our hospital is located. Terrier breeds, especially West Highland White Terrier, and poodles have been over-represented in studies of dogs with lodged EFBs $[1,6$, $15,21,24]$.

In addition to its retrospective design, this study had several other limitations. Dogs with incomplete treatment and follow-up records were excluded, which may have introduced a selection bias. In addition, the present study did not compare the severity of complications. Moreover, despite EFBs being three-dimensional structures, this study did not evaluate their volumes. Nevertheless, the length of the long- est side that affects the upper and lower esophagus may be an important determinant of damage to the muscular layer. To account for differences in esophageal diameters among breeds, the dimension of the EFBs should have been normalized to the dimensions of the esophagus in individual animals. Moreover, longer-term follow-up evaluations are required

In conclusion, this study found that older age, a longer duration of impaction, and a larger dimension of EFBs may be risk factors for the development of complications in dogs with EFBs. Although EFBs are common and comparatively easy to diagnose, based on history, radiography, and clinical signs, complications of EFBs can easily be overlooked. Risk factors for complications identified in this study may be valuable for predicting prognosis.

\section{Acknowledgments}

The authors would like to thank all the owners of the dogs included in this study. This research was supported by the Basic Science Research Program through the National Research Foundation of Korea, which was funded by the Ministry of Science, ICT and Future Planning (NRF2016R1A1A1A05005395).

\section{References}

1. Houlton JE, Herrtage ME, Taylor PM, Watkins SB. Thoracic oesophageal foreign bodies in the dog: a review of ninety cases. J Small Anim Pract 1985;26:521-536.

2. Michels GM, Jones BD, Huss BT, Wagner-Mann C. Endoscopic and surgical retrieval of fishhooks from the stomach and esophagus in dogs and cats: 75 cases (19771993). J Am Vet Med Assoc 1995;207:1194-1197.

3. Pearson H. Symposium on conditions of the canine oesophagus. I. Foreign bodies in the oesophagus. J Small Anim Pract 1966;7:107-116.

4. Washabau RJ. Esophagus. In: Washabau RJ, Day MJ (eds.). Canine and Feline Gastroenterology. pp. 570-605, Elsevier, Philadelphia, 2012.

5. Sale CS, Williams JM. Results of transthoracic esophagotomy retrieval of esophageal foreign body obstructions in dogs: 14 cases (2000-2004). J Am Anim Hosp Assoc 2006;42:450456.

6. Spielman BL, Shaker EH, Garvey MS. Esophageal foreign body in dogs: a retrospective study of 23 cases. J Am Anim Hosp Assoc 1992;28:570-574.

7. Radlinsky MA. Surgery of digestive system. In: Fossum TW, Dewey CW, Horn CV, Johnson AL, MacPhail CM, Radlinsky MA, Schulz KS, Willard MD (eds.). Small Animal Surgery. 4th ed. pp. 387-583, Elsevier, Philadelphia, 2013.

8. Juan RG, Uzma DS. Foreign body removal. In: Douglas GA (ed.). Upper Endoscopy for GI Fellows. pp. 31-42, Springer, Gewerbestrasse, 2017.

9. King JM. Esophageal foreign body and aortic perforation in a dog. Vet Med 2001;96:828.

10. Kyles AE. Esophagus. In: Slatter D (ed.). Textbook of Small Animal Surgery. 3rd ed. pp. 573-592, Saunders, Philadelphia, 2003. 
11. Juvet F, Pinilla M, Shiel RE, Mooney CT. Oesophageal foreign bodies in dogs: factors affecting success of endoscopic retrieval. Ir Vet J 2010;63:163-168.

12. Rousseau A, Prittie J, Broussard JD, Fox PR, Hoskinson J. Incidence and characterization of esophagitis following esophageal foreign body removal in dogs: 60 cases (19992003). J Vet Emerg Crit Care 2007;17:159-163.

13. Thompson HC, Cortes Y, Gannon K, Bailey D, Freer S. Esophageal foreign bodies in dogs: 34 cases (2004-2009). J Vet Emerg Crit Care (San Antonio) 2012;22:253-261.

14. Moore AH. Removal of oesophageal foreign bodies in dogs: use of the fluoroscopic method and outcome. J Small Anim Pract 2001;42:227-230.

15. Ryan WW, Greene RW. The conservative management of esophageal foreign bodies and their complications: a review of 66 cases in dogs and cats. J Am Anim Hosp Assoc 1975;11:243-249.

16. Brisson BA, Wainberg SH, Malek S, Reabel S, Defarges A, Sears WC. Risk factors and prognostic indicators for surgical outcome of dogs with esophageal foreign body obstructions. J Am Vet Med Assoc 2018;252:301-308.

17. Sung SH, Jeon SW, Son HS, Kim SK, Jung MK, Cho CM, Tak WY, Kweon YO. Factors predictive of risk for complications in patients with oesophageal foreign bodies. Dig Liver Dis 2011;43:632-635.
18. Guilford WG, Strombeck DR. Diseases of swallowing. In: Strombeck DR (ed.). Strombeck's Small Animal Gastroenterology. 3rd ed. pp. 211-238, WB Saunders, Philadelphia, 1996.

19. Gregersen H, Pedersen J, Drewes AM. Deterioration of muscle function in the human esophagus with age. Dig Dis Sci 2008;53:3065-3070.

20. Watrous BJ, Suter PF. Normal swallowing in the dog: a cineradiographic study. Vet Radiol Ultrasound 1979;20:281292.

21. Gianella P, Pfammatter NS, Burgener IA. Oesophageal and gastric endoscopic foreign body removal: complications and follow-up of 102 dogs. J Small Anim Pract 2009;50:649-654.

22. Leib MS, Sartor LL. Esophageal foreign body obstruction caused by a dental chew treat in 31 dogs (2000-2006). J Am Vet Med Assoc 2008;232:1021-1025.

23. Parker NR, Walter PA, Gay J. Diagnosis and surgical management of esophageal perforation. J Am Anim Hosp Assoc 1989;25:587-594.

24. Luthi C, Neiger R. Esophageal foreign bodies in dogs: 51 cases (1992-1997). Eur J Comp Gastroenterol 1998;3:7-11.

25. Zimmer JF. Canine esophageal foreign bodies: endoscopic, surgical, and medical management. J Am Anim Hosp Assoc 1984;20:669-677. 